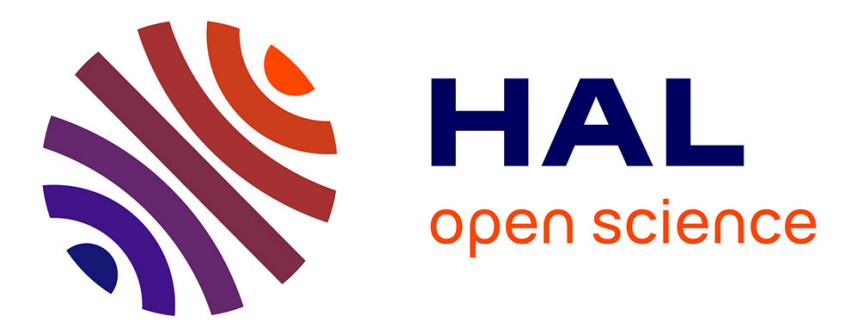

\title{
Un modèle biphasique pour simuler la mise en forme par compression des composites à fibres courtes
}

\author{
Pierre J.J. Dumont, Steven Le Corre, Laurent Orgeas, Denis Favier
}

\section{To cite this version:}

Pierre J.J. Dumont, Steven Le Corre, Laurent Orgeas, Denis Favier. Un modèle biphasique pour simuler la mise en forme par compression des composites à fibres courtes. Revue des composites et des matériaux avancés = Journal of Composite and Advanced Materials, 2002, 12 (3), pp.477-497. 10.3166/rcma.12.477-497. hal-01007421

\section{HAL Id: hal-01007421 \\ https://hal.science/hal-01007421}

Submitted on 16 Jun 2014

HAL is a multi-disciplinary open access archive for the deposit and dissemination of scientific research documents, whether they are published or not. The documents may come from teaching and research institutions in France or abroad, or from public or private research centers.
L'archive ouverte pluridisciplinaire HAL, est destinée au dépôt et à la diffusion de documents scientifiques de niveau recherche, publiés ou non, émanant des établissements d'enseignement et de recherche français ou étrangers, des laboratoires publics ou privés. 


\title{
Un modèle biphasique pour simuler la mise en forme par compression des composites à fibres courtes
}

\section{Pierre Dumont - Steven Le Corre - Laurent Orgéas - Denis Favier}

\author{
Laboratoire Sols - Solides - Structures \\ UMR CNRS 5521 - INPG - UJF \\ BP 53 \\ 38041 Grenoble cedex 9 \\ Pierre.Dumont@hmg.inpg.fr,Steven.Lecorre@hmg.Inpg.fr \\ Laurent.Orgeas@hmg.inpg.fr,Denis.Favier@hmg.inpg.fr
}

RÉSUMÉ. Un formalisme biphasique est proposé pour modéliser la rhéologie des composites fibres de verre - matrice polymère de type SMC ou GMT au cours de leur mise en forme. Ainsi, dans un tel composite, on distingue un premier milieu formé par le réseau de fibres et un second formé par la matrice polymère. L'anisotropie induite par la morphologie du réseau de fibres est simplifiée au cas de l'orthotropie de révolution. Le comportement visqueux non linéaire en loi puissance régit la rhéologie des deux milieux ainsi que leur interaction. Les équations de ce modèle sont simplifiées et adaptées au cas du procédé de compression des SMC; ceci aboutit à la formulation d'un modèle coque, dont les équations sont introduites dans un code éléments finis. Des exemples simples de simulations isothermes permettent alors de souligner l'importance des paramètres " matériau » et "procédé 》 sur le phénomène de redistribution de phases.

ABSTRACT. A two-phase approach is proposed to model the rheology of polymer glass-fiber compounds such as SMC or GMT during processing. Hence, we consider the compounds as formed by two distinct media: the fiber net and the polymer. The anisotropic behaviour, which is induced by the microstructure of the fiber net, is reduced to the simple case of transverse isotropy. The rheology of the two media as well as their interaction follows nonlinear (in power law) viscous behaviours. The equations of this model are simplified to the case of the compression of SMC. This gives the formulation of a shell model whose equations are written into a finite element code. Simple examples of isothermal simulations thus show the strong influence of material and process parameters on the phenomenon of phase separation.

MOTS-CLÉS : SMC, GMT, théorie des mélanges, modèle biphasique coque, anisotropie, loi de filtration, ségrégation.

KEYWORDS: SMC, GMT, theory of mixture, biphasic shell model, anisotropy, filtration law, segregation. 


\section{Introduction}

En raison de leurs bonnes propriétés mécaniques, mais aussi de leur résistance chimique et à la corrosion, les composites à matrice organique chargés de fibres courtes et mis en forme par compression sont largement utilisés par l'industrie automobile ou par l'industrie des composants électriques pour fabriquer des pièces semi-structurelles et légères. C'est dans cette famille de composites que l'on trouve les SMC «Sheet Moulding Compounds» et les GMT «Glass Mat Thermoplastics ». Les SMC et les GMT ont la même morphologie: ils sont fabriqués sous forme de feuilles préimprégnées de 2 à $3 \mathrm{~mm}$ d'épaisseur constituées d'un mat de fibres de verre, enchevêtrées et réparties aléatoirement, noyées dans une matrice polymère. Cette matrice est thermodurcissable pour les SMC et thermoplastique pour les GMT. Après découpe, un empilement de 3 à 10 feuilles de ces matériaux est mis en forme par compression dans un moule. Lors de la phase de remplissage du moule, des phénomènes de redistribution non-uniforme en taux et en orientation des fibres peuvent se produire (Yaguchi et al., 1995). Ces phénomènes qui conditionnent en grande partie aussi bien la tenue mécanique que la géométrie des pièces moulées doivent être maîtrisés.

La plupart des modèles de simulation utilisés pour décrire l'écoulement des matériaux composites à matrice organique tels que les SMC et les GMT au cours de leur mise en forme voient ces matériaux comme des milieux monophasiques, incompressibles et conservant un taux de fibres homogène au cours de leur déformation. Malgré leur intérêt, ces modèles ne peuvent pas décrire les phénomènes de redistribution des fibres, qui se traduisent par des taux de fibres non homogènes à l'intérieur des pièces finies, car l'écoulement de la matrice et des fibres est décrit par un même champ de vitesse.

Afin de décrire ces changements dans la composition du mélange fibres matrice polymère, on adopte ici une modélisation écrite dans le cadre de la théorie des mélanges multiphasés. Ce genre de formalisme a déjà été appliqué avec succès pour prédire les hétérogénéités de répartition se produisant au cours des procédés tels que l'injection de poudres métalliques dans une matrice polymère (Lantéri et al., 1996) (Barrière et al., 2001), l'extrusion de pâtes minérales (Racineux, 1999), ou bien encore les procédés de mise en forme des alliages métalliques à l'état semisolide tels que le thixoformage (Nguyen et al., 1994) (Gebelin, 2000) (Favier et al., 2002).

Dans ce travail, on distingue le milieu formé par le réseau de fibres de celui formé par la matrice polymère. L'anisotropie induite par la morphologie du réseau de fibres est simplifiée au cas de l'orthotropie de révolution. Le comportement visqueux non linéaire en loi puissance régit la rhéologie des deux milieux ainsi que leur interaction. Après un bref rappel du cadre général de la théorie des mélanges, le modèle biphasique "SMC-GMT» est présenté (\$2). Les équations de ce modèle sont alors simplifiées et adaptées au cas du procédé de compression des SMC ; ceci 
aboutit à la formulation d'un modèle coque $(\S 3)$. Les équations de ce modèle coque sont introduites dans un code éléments finis (§4). Des exemples simples de simulations isothermes permettent alors de souligner l'importance des paramètres matériaux et des paramètres procédés sur le phénomène de redistribution de phases $(\S 5)$.

\section{Modélisation biphasique}

L'approche de la théorie des mélanges multiphasés - c'est-à-dire comportant plusieurs constituants non miscibles (Bedford et Drumheller, 1983) - est phénoménologique. Dans cette théorie, chaque constituant est vu comme un milieu continu. Le mélange est décrit comme la superposition de ces milieux qui interagissent entre eux, et qui ont chacun leur propre mouvement. Ainsi à chaque point matériel du mélange correspond un point matériel de chaque constituant. L'écriture des équations de bilan pour les milieux multiphasés a fait l'objet de nombreux travaux théoriques (Truesdell, 1957) (Truesdell et Toupin, 1960) (Truesdell, 1965) (Eringen et Ingram, 1965) (Atkin et Craine, 1976) (Bowen, 1976) (De Boer, 2000). Dans ces travaux, les équations de conservation de masse, de quantité de mouvement et d'énergie sont écrites pour chaque constituant comme pour un milieu continu classique où l'on a néanmoins introduit des termes supplémentaires pour prendre en compte les effets d'échanges de masse, de quantité de mouvement et d'énergie entre les constituants. Le formalisme multiphasique implique de définir des grandeurs pour chaque phase. On note $\Phi_{(\alpha)}$ (avec l'indice $(\alpha)$ en bas) la quantité microscopique, locale ou encore intrinsèque de la grandeur $\Phi$ relative à la phase $\alpha$, car elle correspond à une propriété du matériau dense du constituant $\alpha$. La même grandeur notée $\Phi^{(\alpha)}$ (avec l'indice $(\alpha)$ en haut) est dite macroscopique : elle correspond à la quantité « apparente » de la grandeur $\Phi$ dans le mélange. Dans la théorie des mélanges, chaque volume élémentaire matériel du mélange $\mathrm{d} V$ est occupé, à tout moment, par un volume élémentaire matériel de chaque phase $\alpha$. Une masse volumique macroscopique notée $\rho^{(\alpha)}$ est ainsi associée à chaque phase. La masse $m^{(\alpha)}$ de chaque phase est définie par :

$$
m^{(\alpha)}=\int_{V} \rho^{(\alpha)} \mathrm{d} V
$$

Le volume $\mathrm{d} V^{(\alpha)}$ est défini comme le volume occupé par le constituant $\alpha$ dense de masse volumique microscopique $\rho_{(\alpha)}$ tel que :

$$
m^{(\alpha)}=\int_{V^{(\alpha)}} \rho_{(\alpha)} \mathrm{d} V^{(\alpha)}
$$

La fraction volumique $f^{(\alpha)}$ de la phase $\alpha$ dans le mélange est alors définie par les rapports suivants :

$$
f^{(\alpha)}=\frac{\mathrm{d} V^{(\alpha)}}{\mathrm{d} V} \text { ou } f^{(\alpha)}=\frac{\rho^{(\alpha)}}{\rho_{(\alpha)}}
$$


Si le milieu multiphasique est saturé, la relation $\sum_{\alpha} f^{(\alpha)}=1$ doit être respectée pour tout point et à tout instant.

\subsection{Hypothèses de modélisation dans le cas des SMC ou des GMT}

Les composites à fibres courtes peuvent être vus comme un mélange constitué de deux phases complémentaires poreuses (Le Corre, 2001), une phase pâte $p$ représentant la matrice polymère et une phase fibres $f$ formée par un réseau de fibres.

La modélisation biphasique du comportement des composites comporte les hypothèses générales suivantes :

- les forces volumiques à distance telles que le poids sont considérées comme des quantités négligeables, de même pour les accélérations de chaque phase,

- il n'y a pas d'échange de masse d'un des constituants vers l'autre,

- le milieu est supposé saturé, i.e. $f^{(p)}+f^{(f)}=1$,

- à l'échelle microscopique, les phases pâte et fibres sont incompressibles et les phénomènes de dilatation thermique sont négligés, i.e. $\rho_{(p)}=$ cste et $\rho_{(f)}=$ cste ,

- seules des situations isothermes sont envisagées dans ce travail.

\subsection{Bilans de masses}

Compte tenu des hypothèses précédentes, les deux bilans de masse pour la pâte et les fibres deviennent respectivement :

$$
\begin{aligned}
& \frac{\mathrm{D}^{(p)}}{\mathrm{D} t} f^{(p)}+f^{(p)} \operatorname{div} \underline{\mathbf{v}}^{(p)}=0 \\
& \frac{\mathrm{D}^{(f)}}{\mathrm{D} t} f^{(f)}+f^{(f)} \operatorname{div} \underline{\mathbf{v}}^{(f)}=0
\end{aligned}
$$

où le symbole $\frac{\mathrm{D}^{(\alpha)}}{\mathrm{D} t}$ est la dérivée matérielle en suivant la phase $\alpha$ et où $\underline{\mathbf{v}}^{(p)}$ et $\underline{\mathbf{v}}^{(f)}$ sont respectivement les champs de vitesse macroscopique pâte et fibres. En sommant [4] et [5], on obtient une équation traduisant l'incompressibilité globale avec saturation du mélange :

$$
\operatorname{div}\left(f^{(f)} \underline{\mathbf{v}}^{(f)}+\left(1-f^{(f)}\right) \underline{\mathbf{v}}^{(p)}\right)=0
$$




\subsection{Bilans de quantité de mouvement - Rhéologie des phases pâte et fibres}

Le théorème de la résultante dynamique pour la phase $\alpha$, écrite en négligeant accélération et forces de volume extérieures devient :

$$
\underline{\operatorname{div}} \underline{\underline{\sigma}}^{(\alpha)}+\underline{\hat{\boldsymbol{\pi}}}^{(\alpha)}=\underline{\mathbf{0}}
$$

où $\boldsymbol{\sigma}^{(\alpha)}$ est le tenseur, supposé symétrique, des contraintes partielles associées à chaqüe phase $\alpha$ et où $\hat{\boldsymbol{\pi}}^{(\alpha)}$ représente les forces volumiques exercées par les autres phases présentes dans $V$ sur la phase $\alpha$. Dans le cas des SMC et des GMT, on a

$$
\underline{\hat{\boldsymbol{\pi}}}^{(p)}+\underline{\hat{\boldsymbol{\pi}}}^{(f)}=\underline{\mathbf{0}}
$$

\subsubsection{Décomposition des tenseurs des contraintes partielles}

La théorie des mélanges est un cadre suffisamment large pour proposer une forme très générale du tenseur des contraintes de chacune des phases (Bowen, 1976) (Bedford et Drumheller, 1983) :

$$
\stackrel{\boldsymbol{\sigma}}{=}^{(\alpha)}=\sum_{\beta}^{\beta \neq \alpha} \underline{\boldsymbol{\sigma}}^{(\beta \rightarrow \alpha)}+\underline{\boldsymbol{\sigma}}^{(\alpha) e f f}
$$

où $\boldsymbol{\sigma}^{(\beta \rightarrow \alpha)}$ peut être vu comme l'état de contraintes induit par la phase $\beta$ dans la phase $\alpha$ et où $\boldsymbol{\sigma}^{(\alpha) e f f}$ est le tenseur des contraintes effectives dans la phase $\alpha$, c'està-dire l'état de contraintes que verrait le milieu $\alpha$ en l'absence des autres phases $\beta$. Les expressions des tenseurs $\underline{\underline{\boldsymbol{\sigma}}}^{(f \rightarrow p)}, \underline{\boldsymbol{\sigma}}^{(p) \text { eff }}, \underline{\boldsymbol{\sigma}}^{(p \rightarrow f)}$ et $\underline{\boldsymbol{\sigma}}^{(f) \text { eff }}$ peuvent être établies à partir d'expériences de rhéologie, $d$ 'approches théoriques... Dans ce travail, en se basant sur des expériences isothermes réalisées sur la pâte dense et sur des SMC grâce à un rhéomètre spécialement adapté (Orgéas et al., 2001) (Le Corre et al., 2002) (Dumont et al., 2003), les phases pâte et fibres sont modélisées comme des fluides purement visqueux non-linéaires :

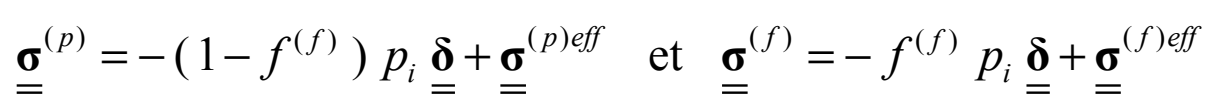

où $p_{i}$ est la pression interstitielle, $\underline{\underline{\boldsymbol{\delta}}}$ le tenseur unité, et où $\underline{\underline{\boldsymbol{\sigma}}}^{(p) e f f}$ et $\underline{\boldsymbol{\sigma}}^{(f) e f f}$ sont les tenseurs des contraintes effectives caractérisant respectivement les efforts visqueux dans le milieu poreux pâte et le milieu poreux fibres. Ce type de décomposition rejoint celle adoptée dans les études de modélisation biphasique des alliages métalliques à l'état semi-solide (Nguyen et al., 1994) (Gebelin, 2000) (Favier et al., 2002) et la mise en forme des matériaux composites (Tucker et Dessenberg, 1994) (Lantéri et al., 1996) (Racineux, 1999) (Barrière et al., 2001). 


\subsubsection{Contrainte effective de la phase pâte $\underline{\underline{\mathbf{\sigma}}}^{(\text {p)eff }}$}

On suppose qu'à l'échelle macroscopique, la pâte n'exerce qu'une action hydrostatique sur le squelette déformable formé par les fibres. Dans ce cas, on écrit :

$$
\underline{\boldsymbol{\sigma}}^{(p) e f f}=\underline{\mathbf{0}}
$$

Ce choix est encore une fois dicté par les expériences réalisées (Orgéas et al., 2001) (Le Corre et al., 2002) (Dumont et al., 2003) où l'on observe que, dans le cas où le taux de fibres massique s'échelonne de 0 à $30 \%$, les viscosités mesurées pour la pâte sont très faibles par rapport à celles mesurées dans les mêmes conditions pour les composites constitués par le mélange de la pâte avec des fibres.

\subsubsection{Contrainte effective dans la phase fibres $\underline{\underline{\boldsymbol{\sigma}}}^{(f) e f f}$}

Dans les composites de type SMC ou GMT, les fibres se situent principalement dans un plan. Dans ce travail, on supposera que l'orientation des fibres dans ce plan est isotrope. Cette microstructure particulière amène à considérer le squelette fibreux comme un milieu orthotrope de révolution. La direction privilégiée de ce milieu est alors celle donnée par un vecteur e perpendiculaire au plan. On introduit alors le tenseur de structure $\underline{\underline{\mathbf{M}}}^{(f)}$ :

$$
\underline{\underline{\mathbf{M}}}^{(f)}=\underline{\mathbf{e}} \otimes \underline{\mathbf{e}}
$$

Ainsi, la forme générale irréductible du comportement effectif du milieu fibres, déduite de résultats courants de la théorie de représentation des fonctions tensorielles isotropes (Boelher, 1987) (Betten, 1988) (Hull et al., 1994), s’écrit :

$$
\begin{aligned}
\underline{\underline{\boldsymbol{\sigma}}}^{(f) e f f} & =\beta_{0} \underline{\underline{\boldsymbol{\delta}}}+\beta_{1} \underline{\underline{\mathbf{M}}}^{(f)}+\beta_{2} \underline{\underline{\mathbf{D}}}^{(f)} \\
& +\beta_{3}(\underline{\underline{\underline{\mathbf{M}}}} \\
& +\beta_{5}(\underline{\underline{\mathbf{M}}} \\
& \left.\left.\underline{\underline{\mathbf{D}}}^{(f)}+\underline{\underline{\mathbf{D}}}^{(f)} \cdot \underline{\underline{\mathbf{D}}}^{(f)}+\underline{\underline{\mathbf{M}}}^{(f)}\right)+\underline{\underline{\mathbf{D}}}^{(f)} \cdot \underline{\underline{\mathbf{D}}}^{(f)} \cdot \underline{\underline{\mathbf{M}}}^{(f)} \cdot \underline{\underline{\mathbf{D}}}^{(f)}\right)
\end{aligned}
$$

où les $\beta_{i}$ sont fonctions des invariants de $\underline{\mathbf{M}}^{(f)}$ et du tenseur vitesse de déformation du milieu fibres $\underline{\underline{\mathbf{D}}}^{(f)}$ dont la liste suit :

$$
\underline{\underline{\mathbf{D}}}^{(f)}: \underline{\underline{\mathbf{D}}}^{(f)},\left(\underline{\underline{\mathbf{D}}}^{(f)} \cdot \underline{\underline{\mathbf{D}}}^{(f)}\right): \underline{\underline{\mathbf{D}}}^{(f)}, \underline{\underline{\mathbf{M}}}^{(f)}: \underline{\underline{\mathbf{D}}}^{(f)} \text { et }\left(\underline{\underline{\mathbf{D}}}^{(f)} \cdot \underline{\underline{\mathbf{M}}}^{(f)}\right): \underline{\underline{\mathbf{D}}}^{(f)}
$$

Dans ce contexte, on suppose que la contrainte $\boldsymbol{\sigma}^{(f) e f f}$ dérive d'un potentiel de dissipation visqueuse $\Omega^{(f) e f f}$, fonction de la vitesse de déformation équivalente effective $D_{e q}^{(f) e f f}$ de la phase fibres et de la fraction volumique $f^{(f)}$. Le tenseur des contraintes effectives de la phase fibres est exprimé en fonction de la vitesse de déformation effective selon la relation : 


$$
\underline{\underline{\boldsymbol{\sigma}}}^{(f) e f f}=\frac{\partial \Omega^{(f) e f f}}{\partial \underline{\underline{\mathbf{D}}}^{(f)}}=\frac{\partial \Omega^{(f) e f f}}{\partial D_{e q}^{(f) e f f}} \frac{\partial D_{e q}^{(f) e f f}}{\partial \underline{\underline{\mathbf{D}}}^{(f)}}=\sigma_{e q}^{(f) e f f} \frac{\partial D_{e q}^{(f) e f f}}{\partial \underline{\underline{\mathbf{D}}}^{(f)}}
$$

où $\sigma_{e q}^{(f) e f f}$ est la contrainte équivalente effective, variable duale de $D_{e q}^{(f) e f f}$. La forme proposée pour $D_{e q}^{(f) e f f}$ est alors la suivante :

$$
D_{e q}^{(f) e f f}{ }^{2}=\alpha_{0}\left(\underline{\underline{\mathbf{D}}}^{(f)}: \underline{\underline{\mathbf{D}}}^{(f)}+\alpha_{1}\left(\underline{\underline{\mathbf{M}}}^{(f)}: \underline{\underline{\mathbf{D}}}^{(f)}\right)^{2}+\frac{1}{2} \alpha_{2}\left(\underline{\underline{\mathbf{D}}}^{(f)} \cdot \underline{\underline{\mathbf{M}}}^{(f)}\right): \underline{\underline{\mathbf{D}}}^{(f)}\right)
$$

avec $\alpha_{0}=\frac{2}{1+2 H^{(f)}}, \alpha_{1}=\left(1+H^{(f)}-2 \frac{1+2 H^{(f)}}{3 L^{(f)}}\right)$ et $\alpha_{2}=2\left(\frac{1+2 H^{(f)}}{3 L^{(f)}}-1\right)$,

où $H^{(f)}(>-0.5)$ et $L^{(f)}(>0)$ sont des fonctions rhéologiques qui caractérisent l'orthotropie de révolution. Elles dépendent de la fraction volumique $f^{(f)}$ et valent 1 dans le cas de l'isotropie. Finalement, le tenseur des contraintes effectives du milieu fibres s'écrit :

$$
\underline{\underline{\boldsymbol{\sigma}}}^{(f) e f f}=\alpha_{0} \eta_{e q}^{(f)}\left(\begin{array}{rl}
\underline{\underline{\mathbf{D}}}^{(f)}+\alpha_{1}\left(\underline{\underline{\mathbf{M}}}^{(f)}: \underline{\underline{\mathbf{D}}}^{(f)}\right) \underline{\underline{\mathbf{M}}}^{(f)} \\
+\frac{1}{2} \alpha_{2}\left(\underline{\underline{\mathbf{M}}}^{(f)} \cdot \underline{\underline{\mathbf{D}}}^{(f)}+\underline{\underline{\mathbf{D}}}^{(f)} \cdot \underline{\underline{\mathbf{M}}}^{(f)}\right)
\end{array}\right)
$$

Dans cette équation, $\eta_{e q}^{(f)}$ est une viscosité équivalente définie comme :

$$
\sigma_{e q}^{(f) e f f}=\eta_{e q}^{(f)} D_{e q}^{(f) e f f} \quad \text { avec } \quad \eta_{e q}^{(f)}=\eta_{0 e q}^{(f)}\left(\frac{D_{e q}^{(f) e f f}}{D_{0}}\right)^{n_{e q}^{(f)}-1}
$$

où $\eta_{0 e q}^{(f)}$ est une viscosité déterminée pour une vitesse de déformation équivalente effective $D_{e q}^{(f) e f f}$ égale à $D_{0}$ (prise ici égale à $1 \mathrm{~s}^{-1}$ ) et $n_{e q}^{(f)}$ est la sensibilité à la vitesse du milieu fibres.

\subsubsection{Modélisation des échanges de quantité de mouvement}

Comme dans le cas des contraintes partielles, une forme très générale est proposée par Bowen (Bowen, 1976) pour modéliser les échanges de quantité de mouvement. Dans le cas particulier de la modélisation des composites à fibres courtes SMC et où la forme de $\underline{\underline{\sigma}}^{(\alpha)}$ est de type [10], on choisira :

$$
\underline{\hat{\boldsymbol{\pi}}}^{(p)}=-p_{i} \underline{\operatorname{grad}} f^{(f)}+\underline{\hat{\boldsymbol{\pi}}}_{b}^{(p)}
$$


Le terme d'échange de quantité de mouvement $\underline{\hat{\boldsymbol{\pi}}}_{b}^{(p)}$ est choisi de sorte que dans une situation où le solide ne se déforme pas et où la pâte s'écoule en régime permanent au travers du squelette fibres avec des nombres de Reynolds locaux négligeables, le bilan de quantité de mouvement pour la pâte se réduise à la loi de filtration dans un milieu poreux d'un fluide en loi puissance de sensibilité à la vitesse $n_{(p)}$ (Christopher et Middleman, 1965) (Kozicki et Tiu, 1988) (Comiti et Renaud, 1989). La structure générale de cette loi de filtration a récemment été obtenue dans le cas d'un milieu poreux anisotrope poreux par passage micro-macro (Auriault et al., 2002). Dans ce travail, il a été montré que lorsqu'un fluide dont la viscosité $\mu_{e q(p)}$ à l'échelle locale était une fonction puissance du taux de cisaillement $\dot{\gamma}_{e q(p)}$, i.e. :

$$
\mu_{e q(p)}=\mu_{0 e q(p)}\left(\frac{\dot{\gamma}_{e q(p)}}{\dot{\gamma}_{0}}\right)^{n_{(p)}-1}
$$$$
\text { avec } \dot{\gamma}_{e q(p)}^{2}=2 \underline{\underline{\mathbf{D}}}_{(p)}: \underline{\underline{\mathbf{D}}}_{(p)}
$$

et où $\mu_{0 e q(p)}, \dot{\gamma}_{0}, n_{(p)}$ sont des constantes

le gradient de pression macroscopique induit par la filtration était une fonction homogène de degré $n_{(p)}$ de la vitesse de filtration macroscopique. Nous proposons ici une forme de $\underline{\hat{\boldsymbol{\pi}}}_{b}^{(p)}$ entièrement compatible avec les travaux d'Auriault et al., et valable dans le cas de l'orthotropie de révolution :

$$
\begin{aligned}
& \underline{\hat{\boldsymbol{\pi}}}_{b}^{(p)}=-\left(1-f^{(f)}\right) \mu_{0 e q(p)} \beta_{0} v_{e q}^{(r)^{n_{(p)}-1}}\left(\beta_{1} \stackrel{\boldsymbol{\delta}}{\underline{\boldsymbol{\delta}}}+\beta_{2} \underline{\underline{\mathbf{M}}}\right) \cdot \underline{\mathbf{v}}^{(r)} \\
& \operatorname{avec} v_{e q}^{(r)^{2}}=\beta_{1} \underline{\mathbf{v}}^{(r)} \cdot \stackrel{\boldsymbol{\delta}}{=} \cdot \underline{\mathbf{v}}^{(r)}+\beta_{2} \underline{\mathbf{v}}^{(r)} \cdot \underline{\underline{\mathbf{M}}}^{(f)} \cdot \underline{\mathbf{v}}^{(r)}
\end{aligned}
$$

où $\underline{\mathbf{v}}^{(r)}$ est la vitesse relative de la pâte par rapport aux fibres, i.e. $\underline{\mathbf{v}}^{(r)}=\underline{\mathbf{v}}^{(p)}-\underline{\mathbf{v}}^{(f)}$, et où les fonctions $\beta_{i}$ dépendent du taux, de l'orientation et de la géométrie des fibres.

Finalement les équations de bilan de quantité de mouvement pour les milieux fibres et pâte s'écrivent :

$$
\begin{aligned}
& -\left(1-f^{(f)}\right) \underline{\operatorname{grad}} p_{i}+\underline{\boldsymbol{\pi}}_{b}^{(p)}=\underline{\mathbf{0}} \\
& -f^{(f)} \underline{\operatorname{grad}} p_{i}+\underline{\operatorname{div}} \underline{\boldsymbol{\sigma}}^{(f) e f f}-\underline{\hat{\boldsymbol{\pi}}}_{b}^{(p)}=\underline{\mathbf{0}}
\end{aligned}
$$

REMARQue. - dans le cas de cinématique monophasique, où $\underline{\mathbf{v}}^{(p)}=\underline{\mathbf{v}}^{(f)}=\underline{\mathbf{v}}$ en tout point, alors la somme des bilans de masses [4] et [5] et la somme des bilans de quantité de mouvement [22] et [23] s'écrivent respectivement : 


$$
\operatorname{div} \underline{\mathbf{v}}=0 \text { et } \underline{\operatorname{div}} \underline{\underline{\sigma}}=\underline{0}
$$

en ayant défini le tenseur de contraintes $\underline{\underline{\sigma}}$ du mélange pâte avec fibres selon :

$$
\begin{aligned}
& \underline{\underline{\boldsymbol{\sigma}}}=\underline{\boldsymbol{\sigma}}^{(p)}+\underline{\boldsymbol{\sigma}}^{(f)}=-p_{i} \underline{\underline{\boldsymbol{\delta}}}+\underline{\boldsymbol{\sigma}}^{(f) e f f} \\
& \operatorname{avec} \stackrel{\boldsymbol{\sigma}}{=}^{(f) e f f}=\alpha_{0} \eta_{e q}^{(f)}\left(\begin{array}{rl}
\underline{\underline{\mathbf{D}}}+\alpha_{1}\left(\underline{\underline{\underline{M}}}^{(f)}: \underline{\underline{\mathbf{D}}}\right) & \underline{\underline{\mathbf{M}}} \\
& \left.+\frac{1}{2} \alpha_{2}\left(\underline{\underline{\mathbf{M}}}^{(f)} \cdot \underline{\underline{\mathbf{D}}}+\underline{\underline{\mathbf{D}}} \cdot \underline{\underline{\mathbf{M}}}^{(f)}\right)\right)
\end{array}\right)
\end{aligned}
$$

Ce modèle monophasique est identique à celui récemment proposé et identifié dans le cas des SMC par Dumont et al. (Dumont et al., 2003).

\section{Application au procédé de compression des composites SMC}

La résolution des équations présentées au paragraphe précédent nécessiterait le calcul de 8 inconnues scalaires $\left(f^{(f)}, \underline{\mathbf{v}}^{(p)}, \underline{\mathbf{v}}^{(f)}, p_{i}\right.$ : voir les équations [6], [7] et [8]) dépendant des trois paramètres d'espace $x_{1}, x_{2}$ et $x_{3}$ ainsi que du temps $t$. Les coûts de calcul d'une pièce réalisée industriellement en SMC seraient alors rédhibitoires étant donné la complexité géométrique des moules généralement utilisés. Heureusement, certains résultats expérimentaux permettent de formuler des hypothèses simplificatrices supplémentaires amenant à l'écriture d'un modèle biphasique coque où le nombre d'inconnues est réduit à 6 .

\subsection{Observations expérimentales de Barone et Caulk (Barone et Caulk, 1985)}

Barone et Caulk ont constaté au cours d'expériences réalisées sur des SMC dans les conditions industrielles du procédé (moule chauffé à $150^{\circ} \mathrm{C}$, échantillons minces de hauteur $h<15 \mathrm{~mm}$, fortes vitesses de compression $\approx 1,75$ à $10 \mathrm{~mm} \mathrm{~s}^{-1}$ ) que l'écoulement des SMC dans le moule suivait une cinématique de type écoulement bouchon : élongation et cisaillement uniquement dans le plan principal des feuilles, pas de cisaillement dans l'épaisseur.

\subsection{Formulation du modèle biphasique coque}

C'est à partir de ce constat expérimental que nous proposons un modèle plaque biphasique d'écoulement des SMC (le plan $\left(\underline{\mathbf{e}}_{1}, \underline{\mathbf{e}}_{2}\right)$ étant le plan des feuilles de $\mathrm{SMC}$ ) facilement adaptable à des géométries de type coques à forts rayons de courbure. Pour le moment, le modèle coque est restreint aux situations isothermes. Il est une extension directe du modèle d'écoulement monophasique de Barone et 
Caulk (Barone et Caulk, 1986). Ainsi, on adopte une hypothèse d'écoulement de type bouchon pour les phases pâte et fibres et l'on suppose aussi que la déformation est homogène dans l'épaisseur $h$ des feuilles de SMC :

$$
\underline{\mathbf{v}}^{(\alpha)}=\left(\begin{array}{c}
v_{1}^{(\alpha)}\left(x_{1}, x_{2}\right) \\
v_{2}^{(\alpha)}\left(x_{1}, x_{2}\right) \\
\frac{\grave{h}}{h} x_{3}
\end{array}\right)_{\underline{\mathbf{e}}_{1}, \underline{\mathbf{e}}_{2}, \underline{\mathbf{e}}_{3}} \quad \Leftrightarrow \underline{\mathbf{D}}^{(\alpha)}=\left(\begin{array}{ccc}
D_{11}^{(\alpha)}\left(x_{1}, x_{2}\right) & D_{12}^{(\alpha)}\left(x_{1}, x_{2}\right) & 0 \\
D_{12}^{(\alpha)}\left(x_{1}, x_{2}\right) & D_{22}^{(\alpha)}\left(x_{1}, x_{2}\right) & 0 \\
0 & 0 & \frac{\dot{h}}{h}
\end{array}\right)_{\underline{\mathbf{e}}_{1}, \mathbf{e}_{2}, \mathbf{e}_{3}}
$$

où, finalement, on contraint les composantes de cisaillement $D_{\beta 3}(\beta \in\{1,2\}) \mathrm{du}$ tenseur cinématique de vitesses de déformation $\underline{\underline{\mathbf{D}}}^{(\alpha)}$ à être nulles. En supposant également que la fraction de fibres $f^{(f)}$ est initialement uniforme dans l'épaisseur des feuilles de SMC, cette cinématique ne peut pas décrire les phénomènes de redistribution en taux de fibres selon $x_{3}$ :

$$
\forall\left(x_{1}, x_{2}, x_{3}\right), \forall t, \quad f^{(f)}\left(x_{1}, x_{2}, x_{3}, t\right)=f^{(f)}\left(x_{1}, x_{2}, t\right)
$$

La condition imposée sur les termes du tenseur de vitesses de déformation de chaque phase $\left(D_{\beta 3}=0, \beta \in\{1,2\}\right)$, nécessite l'adjonction aux lois tensorielles décrivant le comportement de chaque phase d'un terme de réaction arbitraire $T_{\beta 3}^{(\alpha) e f f}$ traduisant l'indéformabilité en cisaillement “ $\beta 3$ ” (Hull et al., 1994) :

$$
\underline{\underline{\boldsymbol{\sigma}}}^{(\alpha)}=-f^{(\alpha)} p_{i} \underline{\underline{\boldsymbol{\delta}}}+T_{\beta 3}^{(\alpha) e f f} \underline{\mathbf{e}}_{\beta} \otimes \underline{\mathbf{e}}_{3}+\underline{\underline{\boldsymbol{\sigma}}}^{(\alpha) e f f}, \quad \beta \in\{1,2\}
$$

En écoulement bouchon, où $\underline{\underline{\mathbf{M}}}^{(f)}=\underline{\mathbf{e}}_{3} \otimes \underline{\mathbf{e}}_{3}$ et $\underline{\mathbf{v}}^{(r)} \cdot \underline{\mathbf{e}}_{3}=0$, la loi d'échanges de quantité de mouvement devient :

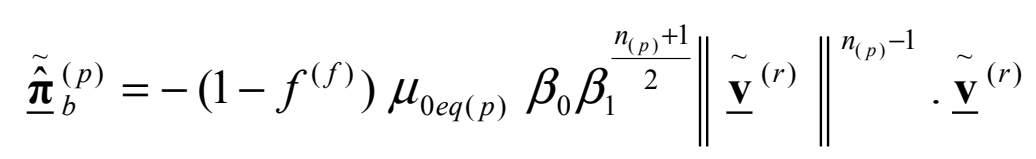

Les quantités vectorielles ou tensorielles surmontées du symbole $\sim$ sont définies seulement dans le $\operatorname{plan}_{n_{(p)}+1}\left(\underline{\mathbf{e}}_{1}, \underline{\mathbf{e}}_{2}\right)$ et ne dépendent que de $x_{1}$ et de $x_{2}$.

Le terme $\beta_{0} \beta_{1}^{\frac{(p)}{2}}$ apparaissant dans l'équation précédente est similaire à la forme proposée par Comiti et Renaud dans le cas de l'écoulement d'un fluide nonnewtonien dans un milieu poreux par (Comiti et al., 1989):

$$
\beta_{0} \beta_{1}^{\frac{n_{(p)}+1}{2}}=\left[\frac{3 n_{(p)}+1}{2 n_{(p)}}\right]^{n_{(p)}} \frac{\left(f^{(f)} \psi\right)^{n_{(p)}+1}}{\left(1-f^{(f)}\right)^{1+2 n_{(p)}}}
$$

où $\psi$ est un paramètre dépendant de la géométrie du poreux. Dans le cas où le fluide filtrant est newtonien, i.e. pour $n_{(p)}=1$, cette expression revient à celle de la perméabilité proposée par Carman-Kozeny (Carman, 1937). 
Pour décrire l'évolution du SMC dans son moule, un point de vue lagrangien est adopté dans l'épaisseur des feuilles SMC (portée par $\underline{\mathbf{e}}_{3}$ ), alors qu'un point de vue eulérien est choisi dans le plan $\left(\underline{\mathbf{e}}_{1}, \underline{\mathbf{e}}_{2}\right)$. Les équations de conservation de la masse et de la quantité de mouvement sont réécrites et simplifiées sous ces conditions. Ainsi, l'équation de conservation de la masse du milieu fibres devient :

$$
\frac{\partial f}{\partial t}^{(f)}+\underline{\operatorname{arad}} f^{(f)} \cdot \underline{\tilde{\mathbf{v}}}^{(f)}+f^{(f)} \tilde{\operatorname{div}} \underline{\tilde{\mathbf{v}}}^{(f)}+f^{(f)} \frac{\dot{h}}{h}=0
$$

L'équation de conservation globale du volume du mélange [6] modifiée s'écrit :

$$
\tilde{\operatorname{div}}\left(f^{(f)} \underline{\mathbf{v}}^{(f)}+\left(1-f^{(f)}\right) \underline{\mathbf{v}}^{(p)}\right)+\frac{\dot{h}}{h}=0
$$

Dans le plan $\left(\underline{\mathbf{e}}_{1}, \underline{\mathbf{e}}_{2}\right)$, les équations de conservation de la quantité de mouvement pour les deux phases sont intégrées dans l'épaisseur $h$ des feuilles SMC et deviennent :

$$
\begin{aligned}
& -\left(1-f^{(f)}\right) \underline{\operatorname{grad}} P_{i}+\underline{\tilde{\mathbf{F}}}_{h}^{(p)}+\underline{\tilde{\mathbf{F}}}_{0}^{(p)}+\underline{\tilde{\mathbf{\Pi}}}_{b}^{(p)}=\underline{\tilde{\mathbf{0}}} \\
& -f^{(f)} \underline{\operatorname{grad}} P_{i}+\underline{\tilde{\mathbf{F}}}_{h}^{(f)}+\underline{\tilde{\mathbf{F}}}_{0}^{(f)}+\underline{\tilde{\mathbf{d i v}}} \underline{\underline{\tilde{\boldsymbol{\Sigma}}}}{ }^{(f) e f f}-\underline{\tilde{\boldsymbol{\Pi}}}_{b}^{(p)}=\underline{\tilde{\mathbf{0}}} \\
& \text { avec } \Sigma_{\beta \lambda}^{(\alpha) e f f}=\int_{0}^{h} \sigma_{\beta \lambda}^{(\alpha) e f f} d x_{3} \text { où }(\beta, \lambda) \in\{1,2\}^{2} \text {, } \\
& \text { et } P_{\mathrm{i}}=h p_{i} \text {, } \\
& \tilde{\Pi}_{\beta}^{(p)}=\int_{0}^{h} \hat{\pi}_{b_{\beta}}^{(p)} d x_{3} \text { où } \beta \in\{1,2\} \\
& \text { et }\left[T_{\beta 3}^{(\alpha) \text { eff }}\right]_{0}^{h}=\left(\underline{\tilde{\mathbf{F}}}_{h}^{(\alpha)}+\underline{\tilde{\mathbf{F}}}_{0}^{(\alpha)}\right) \underline{\mathbf{e}}_{\beta} \text { avec } \beta \in\{1,2\}
\end{aligned}
$$

Les vecteurs $\tilde{\mathbf{F}}_{h}^{(\alpha)}$ et $\underline{\mathbf{F}}_{0}^{(\alpha)}$ permettent ainsi de tenir compte des efforts surfaciques de frottement engendrés par le contact du SMC avec les parois inférieures $\left(x_{3}=0\right)$ et supérieures $\left(x_{3}=h\right)$ du moule. Dans ce travail, on supposera simplement que ces efforts ne s'exercent que sur le milieu fibres et qu'ils sont engendrés par le cisaillement d'une fine couche de pâte lubrificatrice présente entre le SMC et les parois du moule (Cheevers, 1978) (Barone et Caulk, 1986) (Collister et Allen, 1993). Leur expression est : 


$$
\underline{\tilde{\mathbf{F}}}_{h}^{(f)}=\underline{\tilde{\mathbf{F}}}_{0}^{(f)}=-f^{(f)} \mu_{0 e q(p)} \frac{\kappa_{H}}{\kappa_{H 0}}\left(\frac{\underline{\tilde{\mathbf{v}}}^{(f)} \cdot \underline{\mathbf{v}}^{(f)}}{v_{0}{ }^{2}}\right)^{\frac{n_{(p)}-1}{2}} \underline{\tilde{\mathbf{v}}}^{(f)}
$$

où $\kappa_{H}$ est un paramètre à déterminer caractérisant le frottement par rapport à un frottement «caractéristique » $\kappa_{H 0}$ (ici pris égal à 1 ) et une vitesse caractéristique $v_{0}$ de $1 \mathrm{~mm} \mathrm{~s}^{-1}$.

Moyennant un jeu de conditions initiales et de conditions aux limites approprié dans le plan $\left(\underline{\mathbf{e}}_{1}, \underline{\mathbf{e}}_{2}\right)$, le système d'équations constitué de [31], [32] et [33] muni des lois de comportement fibres et pâte, d'interaction fibres-pâte et de frottement SMC-moule, permet le calcul des inconnues $f^{(f)}, P_{i}, \underline{\mathbf{v}}^{(p)}, \underline{\mathbf{v}}^{(f)}$.

\section{Implantation numérique du modèle biphasique coque}

La résolution de l'équation [31] d'évolution de la fraction de fibres $f^{(f)}$ est découplée de celle du système d'équations formé par [32] et [33] qui décrivent l'écoulement du milieu biphasique. Dans ce travail, seules des simulations réalisées à partir d'un champ de fraction de fibres connu a priori sont présentées. La résolution de [31] n'est pas effectuée, mais son utilisation permettra de donner l'évolution instantanée du champ $f^{(f)}$ (voir dans la suite l'équation [44]).

Le problème d'écoulement du milieu biphasique, qui comporte trois champs inconnus $P_{i}, \underline{\mathbf{v}}^{(p)}, \underline{\mathbf{v}}^{(f)}$ est résolu par une formulation mixte « vitesses-pression » de la méthode des éléments finis (Heuzey et al., 2001) sur un domaine $\Omega$ (de bord $\partial \Omega$ ). Pour bien poser le problème, il faut d'abord spécifier les conditions aux limites pour chaque phase sur le bord $\partial \Omega$. Celui-ci se décompose en deux parties disjointes $\partial_{1} \Omega^{(p)}$ et $\partial_{2} \Omega^{(p)}$ pour la pâte (respectivement $\partial_{1} \Omega^{(f)}$ et $\partial_{2} \Omega^{(f)}$ pour les fibres).

- $\quad$ Sur $\partial_{l} \Omega^{(\alpha)}$, on impose une condition cinématique telle que : $\underline{\tilde{\mathbf{v}}}^{(\alpha)} \cdot \underline{\tilde{\mathbf{n}}}=0$ où $\underline{\tilde{\mathbf{n}}}$ est la normale extérieure au domaine $\Omega\left(\partial_{l} \Omega^{(\alpha)}\right.$ est la partie du bord $\partial \Omega$ en contact avec les parois du moule).

- $\quad$ Sur $\partial_{2} \Omega^{(\alpha)}$, on impose $\underline{\underline{\tilde{\Sigma}}}^{(\alpha)} \cdot \underline{\tilde{\mathbf{n}}}=\underline{\tilde{\mathbf{0}}}\left(\partial_{2} \Omega^{(\alpha)}\right.$ représente une surface libre pour la phase $\alpha$ ).

La formulation variationnelle du système est alors obtenue après multiplication de chaque équation par une fonction test et intégration sur le domaine $\Omega$. Elle s'écrit :

Trouver $P_{i}, \underline{\tilde{\mathbf{v}}}^{(p)}, \underline{\tilde{\mathbf{v}}}^{(f)}$ telles que:

$$
\forall \underline{\tilde{\mathbf{w}}}^{(p)^{*}} \in V_{0}^{(p)}=\left\{\tilde{\mathbf{w}}^{(p)^{*}} \text { régulière } \mid \underline{\tilde{\mathbf{w}}}^{(p)^{*}} \cdot \underline{\tilde{\mathbf{n}}}=\tilde{\tilde{\mathbf{0}}} \operatorname{sur} \partial_{1} \Omega^{(p)}\right\}
$$




$$
\begin{aligned}
& -\int_{\Omega}\left(1-f^{(f)}\right) \underline{\operatorname{grad}} P_{i} \cdot \underline{\tilde{\mathbf{w}}}^{(p)^{*}} \mathrm{~d} \Omega+\int_{\Omega} \underline{\tilde{\boldsymbol{\Pi}}}_{b}^{(p)} \cdot \underline{\tilde{\mathbf{w}}}^{(p)^{*}} \mathrm{~d} \Omega=0 \\
& \forall \underline{\tilde{\mathbf{w}}}^{(f)^{*}} \in V_{0}^{(f)}=\left\{\underline{\tilde{\mathbf{w}}}^{(f)^{*}} \text { régulière } \mid \underline{\tilde{\mathbf{w}}}^{(f)^{*}} \cdot \underline{\tilde{\mathbf{n}}}=\underline{\mathbf{0}} \operatorname{sur} \partial_{1} \Omega^{(f)}\right\}, \\
& \int_{\Omega} \underline{\tilde{\operatorname{div}}} \underline{\tilde{\Sigma}}^{(f) e f f} \cdot \underline{\tilde{\mathbf{w}}}^{(f)^{*}} \mathrm{~d} \Omega-\int_{\Omega} f^{(f)} \underline{\underline{\operatorname{grad}}} P_{i} \cdot \underline{\tilde{\mathbf{w}}}^{(f)^{*}} \mathrm{~d} \Omega \\
& +\int_{\Omega}\left(\underline{\tilde{\mathbf{F}}}_{h}^{(f)}+\underline{\tilde{\mathbf{F}}}_{0}^{(f)}\right) \cdot \underline{\tilde{\mathbf{w}}}^{(f)^{*}} \mathrm{~d} \Omega-\int_{\Omega} \underline{\tilde{\boldsymbol{\Pi}}}_{b}^{(f)} \cdot \underline{\tilde{\mathbf{w}}}^{(f)^{*}} \mathrm{~d} \Omega=0 \\
& \forall p^{*} \in P=\left\{p^{*} \text { régulière }\right\} \\
& \int_{\Omega}\left(\tilde{\operatorname{div}}\left(f^{(f)} \underline{\tilde{\mathbf{v}}}^{(f)}+\left(1-f^{(f)}\right) \underline{\mathbf{v}}^{(p)}\right)+\frac{\dot{h}}{h}\right) p^{*} \mathrm{~d} \Omega=0
\end{aligned}
$$

Cette formulation a été implantée dans le logiciel FEMLAB ${ }^{\circledR}$ (FEMLAB, 2002) où sa discrétisation est réalisée au moyen d'éléments P2-P1. L'approximation de chacune des vitesses pâte et fibres est quadratique (P2). La pression est quant à elle linéaire $(\mathrm{P} 1)$, mais discontinue d'un élément à l'autre. La résolution du système d'équations non-linéaires obtenu est réalisée directement en combinant la méthode de linéarisation de Newton (Ciarlet, 1998) et l'algorithme LU (Hernell, 2001).

\section{Résultats de simulations numériques de remplissage isothermes}

\subsection{Matériau, fonctions et paramètres rhéologiques}

C'est en se basant sur les expériences de rhéométrie réalisées en laboratoire (Orgéas et al., 2001) (Le Corre et al., 2002) (Dumont et al., 2003) sur un SMC de formulation Low Profile fourni par la société Mecelec Composite - France qu'une grande partie des paramètres constitutifs du modèle ainsi formulé ont été identifiés.

Ainsi, la viscosité de la pâte suit l'évolution donnée par l'équation [20] avec une sensibilité à la vitesse de déformation $n_{(p)}$ qui vaut 0.58 à $T=296 \mathrm{~K}$ et 0.8 à $T=353 \mathrm{~K}$. La viscosité $\mu_{0 e q(p)}$ à $\dot{\gamma}_{0}$ (choisie à $1 \mathrm{~s}^{-1}$ ) suit une évolution de la forme :

$$
\mu_{0 e q(p)}=\mu_{00 e q(p)} e^{-b_{(p)}\left(\frac{1}{T_{r}}-\frac{1}{T}\right)}
$$

où $\mu_{00 e q(p)}=0.06 \mathrm{MPa} \mathrm{s}, T_{r}=296 \mathrm{~K}$ et $b_{(p)}=4600 \mathrm{~K}$. La viscosité du SMC $\eta_{e q}^{(f)}$ suit l'évolution donnée par l'équation [18] avec par exemple $n^{(f)}=0.44$ de $T_{r}$ à $T=353 \mathrm{~K}$ pour $f^{(f)}=0.235$. Comme pour la pâte, on a : 


$$
\eta_{0 e q}^{(f)}=\eta_{00 e q(p)} e^{-b^{(f)}\left(\frac{1}{T_{r}}-\frac{1}{T}\right)}
$$

où $b^{(f)}=4900 \mathrm{~K}$, et où :

$$
\eta_{00 e q}^{(f)}=0.18\left(1+98 f^{(f)}+980 f^{(f)^{2}}\right)\left(2 \frac{1+H^{(f)}}{1+2 H^{(f)}}\right)^{-\frac{n_{e q}^{(f)}+1}{2}}
$$

Enfin, dans une gamme de fractions volumiques de fibres comprises entre 3.5\% et $23.5 \%$ (taux industriel), les fonctions rhéologiques $H^{(f)}$ et $L^{(f)}$ s'écrivent respectivement :

$$
\begin{aligned}
& H^{(f)}=\frac{1+98 f^{(f)}+980 f^{(f)^{2}}}{0.5+67 f^{(f)}+670 f^{(f)^{2}}}-1 \\
& L^{(f)}=\frac{1+2 H^{(f)}}{6\left(1+H^{(f)}\right)}\left(\frac{1+98 f^{(f)}+980 f^{(f)^{2}}}{0.33+3 f^{(f)}}\right) \frac{2}{n_{e q}^{(f)+1}}
\end{aligned}
$$

Seuls les paramètres $\psi$, régissant l'interaction fibres-pâte, et $\kappa_{H}$, régissant l'interaction SMC-moule n'ont pas été identifiés. D'autres expériences doivent être menées pour estimer plus précisément leur valeur. Dans ce travail, on se contentera d'analyser leur influence sur les résultats.

\subsection{Essai de compression en canal isotherme}

Pour souligner l'importance des paramètres «procédé » et «matériau » sur le phénomène de redistribution de phases, on considère le problème très simple de la compression en canal, ou compression en déformation plane ( $c f$. figure 1). Un échantillon de SMC parallélépipédique de dimensions $L \times l \times h$, à une température homogène $T$, avec un taux volumique de fibres initial et uniforme $f^{0(f)}$, dont les fibres sont réparties aléatoirement dans le plan $\left(\underline{\mathbf{e}}_{1}, \underline{\mathbf{e}}_{2}\right)$, de longueur $x_{1 \max }=L$ de largeur $x_{2 \max }=l$ et de hauteur $x_{3 \max }=h$, est comprimée en déformation plane, i.e. sous un champ cinématique tel que $D_{22}^{(\alpha)}=0$, sous une vitesse de déformation de compression $\dot{h} / h$ telle que $\dot{h} / h=D_{33}^{(\alpha)}=D_{33}$.

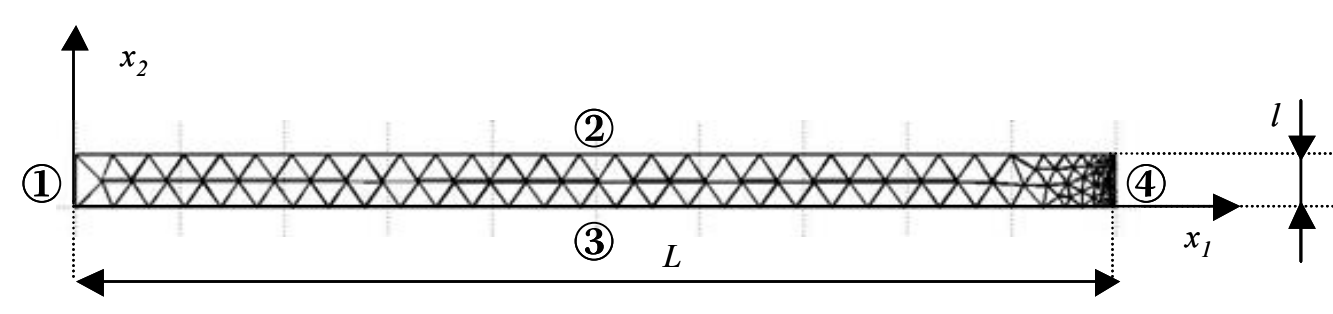

\section{Conditions aux limites}

- Bords (1), (2) et (3) : glissement à la paroi, $\underline{\tilde{\mathbf{v}}}^{(p)} \cdot \underline{\tilde{\mathbf{n}}}=0$ et $\underline{\mathbf{v}}^{(f)} \cdot \underline{\tilde{\mathbf{n}}}=0$.

- Bord (4) : front de matiere, $\underline{\underline{\Sigma}}^{(p)} \underline{\tilde{\mathbf{n}}}=\underline{\tilde{\mathbf{0}}}$ et $\underline{\underline{\underline{\Sigma}}} \underline{\tilde{\Sigma}}^{(f)} \cdot \underline{\tilde{\mathbf{n}}}=\underline{\tilde{\mathbf{0}}}$. 
Figure 1. Géométrie de référence, conditions aux limites et maillage de l'essai de compression en canal.

On définit un essai dit de référence pour les valeurs de paramètres suivantes : $T=353 \mathrm{~K}, D_{33}=-1 \mathrm{~s}^{-1}, f^{0(f)}=0.235, \psi=1$ et $\kappa_{H}=0$. La géométrie de référence est celle d'un parallélépipède de $500 \times 10 \times 10 \mathrm{~mm}^{3}$. Sur la figure 2 , on précise les conditions aux limites ainsi que le maillage P2P1 utilisés. Dans cet essai, les paramètres «procédé » $T, D_{33}$ et $L$ et le paramètre matériau $f^{0(f)}$ ont des valeurs moyennes rencontrées industriellement. Les valeurs du paramètre matériau $\psi$ et du frottement $\kappa_{H}$ sont fixées par défaut. Les différents essais présentés dans la suite se basent sur cette situation de référence pour montrer l'influence de chaque paramètre pris individuellement (seules la hauteur $h$ et la largeur des échantillons resteront inchangées et fixées à $10 \mathrm{~mm}$ ). Afin de pouvoir caractériser le phénomène de redistribution des phases, nous introduisons un taux local de ségrégation noté $D_{\text {seg }}$ tel que :

$$
D_{\text {seg }}=\frac{1}{\left|D_{33}\right|} \frac{\mathrm{D}^{(f)}}{\mathrm{D} t} f^{(f)}=-f^{(f)} \frac{\operatorname{div} \underline{\mathbf{v}}^{(f)}}{\left|D_{33}\right|}
$$

Plus $D_{\text {seg }}$ est grand et plus le phénomène de redistribution du taux de fibres ou ségrégation est important. Inversement, un comportement monophasique est enregistré lorsque $D_{\text {seg }}=0$.

5.2.1. Influence des paramètres « procédé » $\left(T, D_{33}\right.$ et $\left.L\right)$
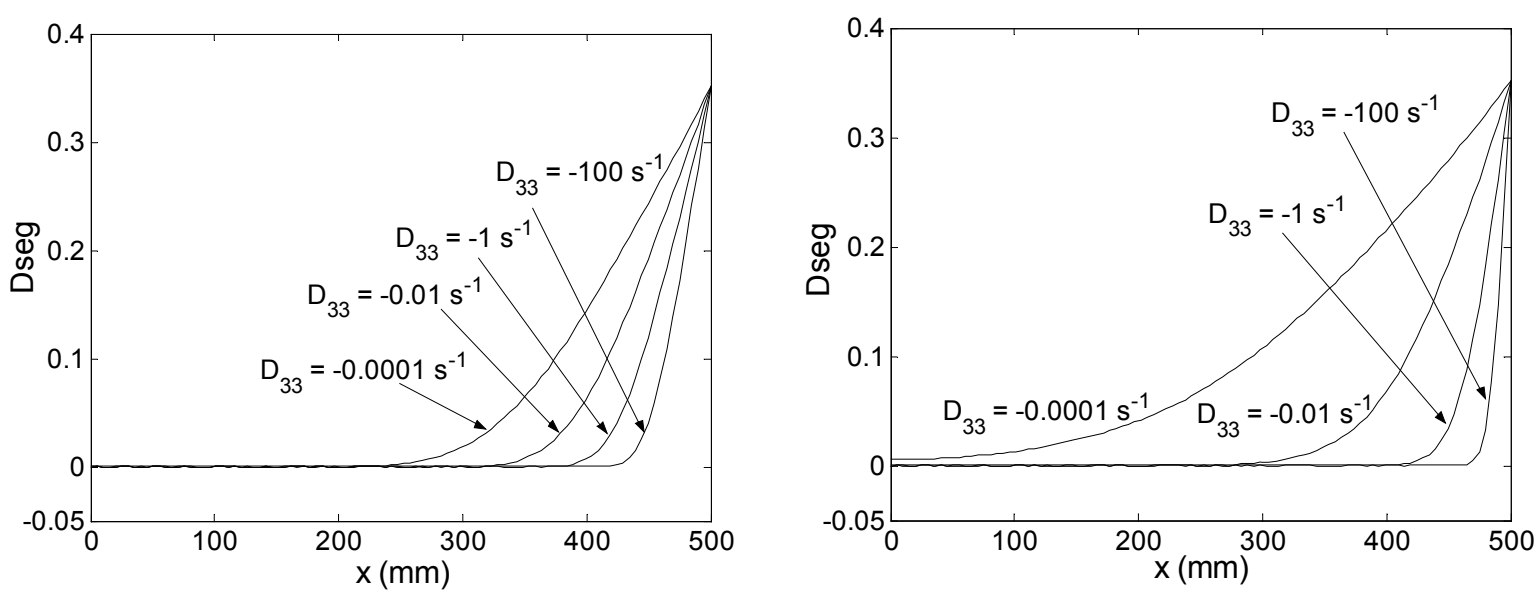

Figure 2. Influence de la vitesse de déformation de compression $D_{33}$ sur le taux de

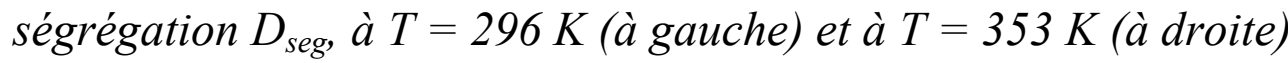

La figure 2 montre l'influence de la vitesse de déformation de compression $D_{33}$ sur le taux de ségrégation pour $T=296 \mathrm{~K}$ et pour $T=353 \mathrm{~K}$, les autres paramètres correspondant à la situation de référence. Typiquement, les tendances observées pour ces deux températures montrent que plus la vitesse de déformation de compression est faible, plus le phénomène de séparation de phase est important, 
c'est-à-dire qu'une grande quantité de pâte va être expulsée du milieu fibres. Inversement, à fort taux de déformation, le milieu tend plutôt à adopter un comportement de type monophasique. On observe aussi que ce changement de régime biphasique $\leftrightarrow$ monophasique est beaucoup plus marqué à haute température $(353 \mathrm{~K})$. Ceci est essentiellement imputable à l'augmentation du contraste de rhéologie à haute température entre le milieu fibres et le milieu pâte (Geindreau et Auriault, 1999) : $n^{(f)}=0.44$ et $n_{(p)}=0.58$ à l'ambiante, alors que $n^{(f)}=0.44$ et $n_{(p)}=0.8$ à $T=353 \mathrm{~K}$.
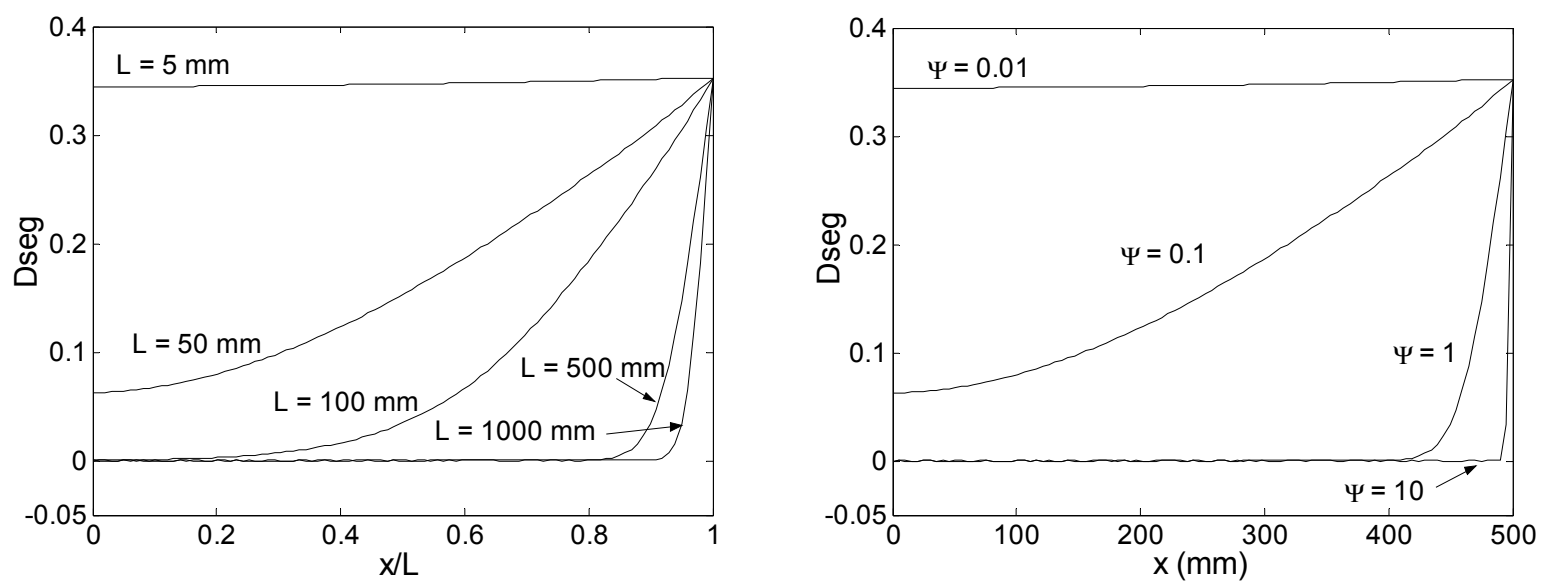

Figure 3. Influence de la longueur $L d u$ domaine de compression (à gauche) et du paramètre $\psi\left(\right.$ à droite) sur le taux de ségrégation $D_{\text {seg }}$
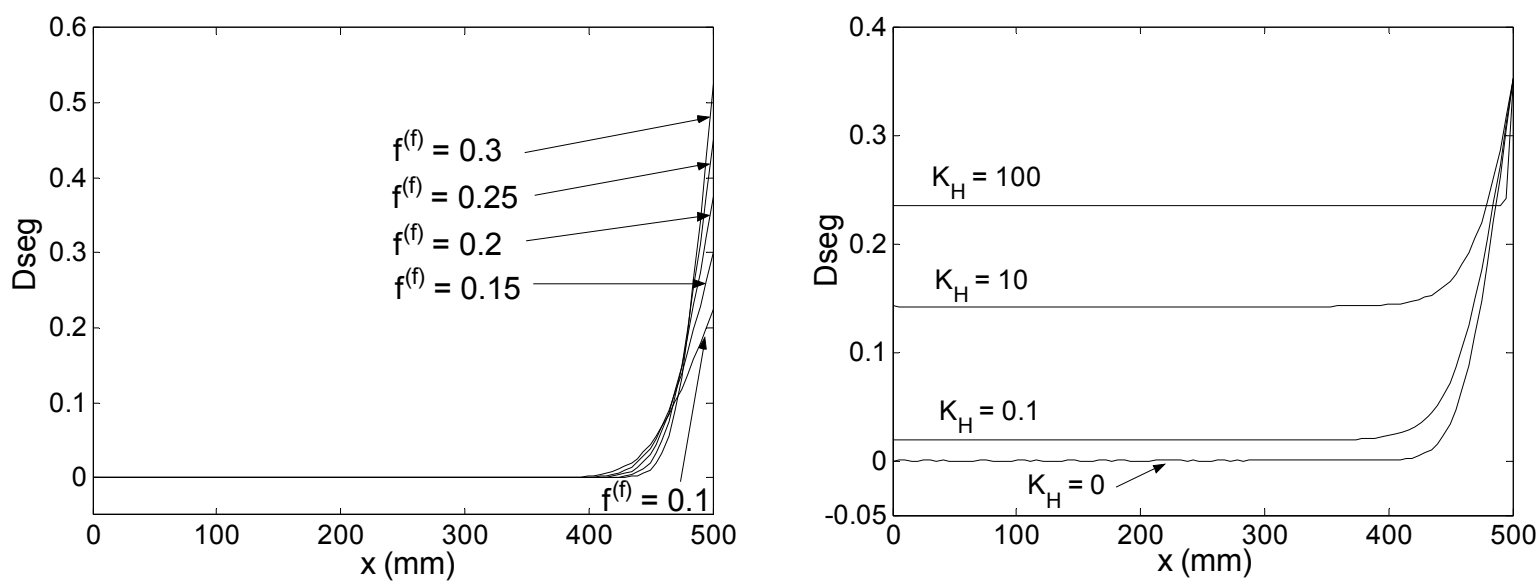

Figure 4. Influence de la fraction volumique de fibres initiale $f^{0(f)}$ (à gauche) et $d u$ paramètre $\kappa_{H}$ (à droite) sur le taux de ségrégation $D_{\text {seg }}$

La figure 3 (à gauche) étudie l'influence de la longueur $L$ de l'échantillon sur $D_{\text {seg }}$. Cette figure montre clairement la très forte influence de $L:$ plus $L$ est grand, et plus le SMC adopte un mode de déformation monophasique. Ce résultat est induit par la nature du terme d'interaction, basé sur la notion de filtration: à même microstructure, il est plus difficile de générer la filtration de la pâte dans un réseau fibreux de grande longueur que dans un réseau fibreux plus court. 


\subsubsection{Influence des paramètres microstructuraux $\left(f^{0(f)}\right.$ et $\left.\psi\right)$}

La figure 3 (à droite) représente l'évolution de $D_{\text {seg }}$ en fonction du paramètre $\psi$ de la loi d'interaction. Ce paramètre, qui est fonction des caractéristiques géométriques à l'échelle microscopique du réseau fibreux, influe très fortement sur le phénomène de ségrégation. Pour les faibles valeurs de $\psi$, qui tendent à diminuer le terme d'interaction, le comportement biphasique du milieu est fortement marqué ; ainsi, pour $\psi<1$, tout l'échantillon est en mode de déformation biphasique. Par contre, pour les fortes valeurs de ce paramètre, le comportement est plutôt monophasique : l'expulsion de la pâte du réseau fibreux est entravée.

La figure 4 (à gauche) montre quant à elle que $D_{\text {seg }}$ est relativement peu affecté par la fraction volumique initiale de fibres $f^{0(f)}$. Ce résultat n'est pas intuitif : il résulte de la compétition à l'évidence équilibrée entre le renforcement avec $f^{0(f)}$ des propriétés rhéologiques du réseau de fibres et l'augmentation du terme d'interaction lorsque $f^{0(f)}$ augmente.

\subsubsection{Influence du frottement moule-SMC $\left(\kappa_{H}\right)$}

Le paramètre $\kappa_{H}$ caractérise le frottement intervenant au niveau du contact entre le réseau de fibres et le moule. Son influence sur $D_{\text {seg }}$ est illustrée sur la figure 4 (à droite). Par rapport à la situation de référence idéale, où $\kappa_{H}=0$, on remarque que l'augmentation de $\kappa_{H}$ induit un accroissement significatif et homogène de $D_{\text {seg }}$ dans l'échantillon: le frottement diminue la déformation du réseau fibreux, si bien que l'expulsion de pâte est facilitée.

REMARQUE. - Une augmentation plus prononcée de $\kappa_{H}$ conduirait à négliger le terme $\tilde{\mathbf{d i v}} \underline{\tilde{\boldsymbol{\Sigma}}^{(f) e f f}}$ par rapport aux termes $\underline{\mathbf{F}}_{h}^{(f)}+\underline{\mathbf{F}}_{0}^{(f)}$ dans l'équation [33] : ceci constituerait un modèle d'approximation de type couche mince (Barone et Caulk, 1986) formulé dans un cadre biphasique.

\section{Conclusion}

Pour modéliser les phénomènes de redistribution en taux de fibres qui se produisent lors de la mise en forme par compression des composites à fibres courtes de type SMC ou GMT, nous avons proposé dans ce travail un modèle biphasique basé sur la théorie des mélanges. Ces composites sont ainsi vus comme la superposition de deux milieux poreux purement visqueux et non-linéaires qui interagissent entre eux : le milieu pâte et le milieu fibres.

Dans ce cadre, le réseau de fibres est vu comme un corps visqueux non linéaire (loi puissance), compressible et orthotrope de révolution. Un modèle rhéologique capable de rendre compte de ces phénomènes est proposé et écrit sous sa forme intrinsèque (utilisation de la représentation des fonctions tensorielles isotropes). Ses paramètres constitutifs ont été entièrement déterminés expérimentalement. Les échanges de quantité de mouvement se produisant entre les milieux fibres et pâtes 
ont été modélisés à partir du problème fondamental de la filtration d'un fluide non newtonien en loi puissance dans un milieu poreux rigide anisotrope.

Ce modèle a été simplifié au cas particulier du procédé de compression des $\mathrm{SMC}$ : un modèle biphasique coque basé sur l'hypothèse d'écoulement bouchon a été formulé, il se restreint pour le moment à des situations isothermes. Le modèle coque, qui permet en outre de tenir compte des frottements SMC-moule, a été intégré dans un code $\mathrm{EF}$.

Des exemples de simulations basés sur le problème élémentaire de la compression en déformation plane ont permis de souligner l'influence des paramètres "procédé » (longueur des échantillons $L$, vitesse de déformation de compression $D_{33}$, température de moulage $T$ ), «matériau » (fraction volumique de fibres $f^{(f)}$, coefficient d'interaction $\psi$ ) et de « contact 》 (coefficient de frottement $\kappa_{H}$ ) sur le phénomène de redistribution en taux de fibres. Les résultats présentés dans ce travail ne constituent que des tendances qualitatives. Ils pourront être utilisés à des fins plus prédictives une fois que les coefficients $\psi$ et $\kappa_{H}$ auront été identifiés. Ce travail est actuellement en cours dans le cas du procédé de compression des SMC.

Les simulations présentées sont basées sur la seule résolution du problème d'écoulement vitesses-pression du milieu biphasique. Plusieurs extensions de ce modèle sont en actuellement en cours de réalisation :

- couplage de la résolution du problème d'écoulement vitesses-pression avec celle du problème de transport de la fraction de fibres,

- prise en compte de la thermique,

- modification des lois de comportement et d'interaction pour tenir compte de l'évolution de l'orientation des fibres au cours de l'écoulement.

Ces extensions sont réalisées dans une maquette numérique industrielle développée en collaboration avec Renault S.A. (poursuite du projet Rhône-Alpes «Action composites SMC-BMC », 1998-2001).

\section{Bibliographie}

Aktin R.J., Craine R.E., J. Inst. Maths. Applics., vol. 17, 1976, p. 153-207.

Auriault J.-L., Royer P., Geindreau C., "Filtration law for power-law fluids in anisotropic porous media", Int. J. Eng. Sci., vol. 40, 2002, p. 1151-1163.

Barone M., Caulk D., "The effect of deformation and thermoset cure on heat conduction in a chopped-fiber reinforced polyester during compression molding", Int. J. of Heat \& Mass Transfer, vol. 22, nº 7, 1979, p. 1021-1031.

Barone M.R., Caulk D.A., "Kinematics of flow in sheet molding compounds", Polymer Composites, vol. 6, n², 1985, p. 105-109. 
Barone M.R., Caulk D.A., "A model for the flow of a chopped fiber reinforced polymer compound in compression molding”, J. Applied Mechs., vol. 53, 1986, p. 361-370.

Barrière T., Renault D., Gélin J.-C., "Experimental and numerical analysis of the effects of process parameters on the properties of components in metal injection molding", $J$. phys., $I V ., 2001$, vol. 84.

Bedford A., Drumheller D.S., "Theories of immiscible and structured mixtures", Int. J. Eng. Sci., vol. 21, n8, 1983, p. 863-960.

Betten J., "Applications of tensor functions to the formulation of yield criteria for anisotropic materials", Int. J. Plasticity, vol. 4, 1988, p. 29-46.

Boehler J.-P., "Application of tensor functions in solid mechanics”, In : Boehler J.-P. (Ed.), CISM Courses and Lectures, $N^{\circ} 292 ., 1987$, Springer Verlag, Wien, NY.

Bowen, "Continuum physics III : mixtures and EM field theories", vol. III, 1976, dans Eringen A.C., Academic Press, New York, p. 1-127.

Carman P.C., "Fluid flow through granular beds", Trans. Institute Chem. Eng., 1937, p. 150166.

Cheevers G., Journal of Coating Technology, vol. 50, 1978, p. 36.

Christopher R. H., Middleman S., "Power-law flow through a packed tube", Ind. Eng. Chem. Fundamentals, vol. 4, 1965, p. 422-426.

Ciarlet P.G., «Introduction à l'analyse numérique matricielle », 1998, Dunod, Paris, p.158166.

Collister J., Allen P., "Sheet molding compound rheology", In : « Sheet molding compounds science and technology », 1993, Ed. Kia H.G., Hanser Publisher, Munich.

Comiti J., Renaud M., “A new model for determining mean structure parameters of fixed beds from pressure drop measurement : application to beds packed with parallelepipedal particules", Chem. Engng. Sci., vol. 7, 1989, p. 1539-1545.

De Boer R., "Contempory progress in porous media theory", Appl. Mech. Rev., 2000, vol. 53, $\mathrm{n}^{\circ} 12$, p. 323

Dumont P., Orgéas L., Le Corre S., Favier D., “Anisotropic viscous behavior of sheet molding compounds (SMC) during compression molding", Int. J. Plasticity, vol. 19, 2003, p. 625-646.

Eringen A.C., Ingram J.D., Int. J. Eng. Sci., vol. 3, 1965, 197-212.

Favier D., Orgéas L., Geindreau C., Bay F., Chastel Y., Bellet M., « Modélisation des phénomènes de ségrégation entre phases liquide et solide» In : "Mise en forme des alliages métalliques à l'état semi-solide », 2002, Ed. M. Suéry, Editions Hermès Science Publications, Paris.

FEMLAB, Reference Manual, version 2.3. http://www.femlab.com.

Gébelin J.-C., Etude expérimentale et simulation numérique du comportement de l'alliage de magnésium AZ91 à l'état semi-solide, Thèse de doctorat, Université Joseph Fourier Grenoble I, 2000. 
Geindreau C., Auriault J.-L., "Investigation of the viscoplastic behaviour for alloys in the semi-solid state by homogenization", Mech. of Mater., vol. 31, 1999, p. 535-551.

Hernell O., "Time-stepping for finite element discretizations of incompressible flow problems in FEMLAB", Master thesis, Royal institute of Technology of Stockholm, 2001.

Heuzey M.-C., Fortin A., Dealy J., «Fluides viscoélastiques : modélisation numériques et mesures expérimentales », Revue européenne des éléments finis, vol. 10, n5, 2001, p. 513-537.

Hull B.D., Rogers T.G., Spencer A.J.M., "Theoritical analysis of forming flows fibre-resin systems" In : Advani S.G. (Ed) "Flow and rheology in polymer composites manufacturing", 1994, Elsevier Science B.V.

Lantéri B., Burlet H., Poitou A., Campion I., "Powder injection molding : an original simulation paste flow", Eur. J. of Mech. A. Solids., vol. 22 , n 7, 1996, p. 943-968.

Kozicki W., Tiu C., "A unified model for non-Newtonian flow in packed beds and porous media”, Rheol. Acta, vol. 27, 1988, p. 31-38.

Le Corre S., Etude de la mise en forme par compression des sheet molding compounds (SMC), Thèse de doctorat, Université Joseph Fourier - Grenoble I, 2001.

Le Corre S., Orgéas L., Favier D., Tourabi A., Maazouz A., Venet C., "Shear and compression behavior of sheet molding compounds", Composites Science and Technology, vol. 62, 2002, p. 571-577.

Orgéas L., Le Corre S., Dumont P., Favier D., Tourabi A., "Influence of fibre volume fraction and mechanical loading on the flow behaviour of sheet molding compounds (SMC)", In : Habraken A.M. (Ed) "The $4^{\text {th }}$ international ESAFORM conference on material forming”; Liège, 23-25 avril 2001.

Nguyen T.G., Favier D., Suéry M., "Theoretical and experimental study of the isothermal mechanical behavior of alloys in the semi-solid state", Int. J. Plasticity, vol. 10, n6, 1994, p. 663-693.

Truesdell C., Rend. Acad. Lincei, vol. 8, n²2, 1957, p. 158-166.

Truesdell C., Toupin R., dans S. Flugge, Encyclopedia of Physics, vol. III/1, 1960, Springer Verlag, Berlin, p. 226

Truesdell C., "Continuum mechanics II : the rational mechanics of materials", 1965, Editions Gordon \& Breach, NY.

Tucker C.L., Dessenberger R.B., "Flow and rheology in polymer composites manufacturing", In : Advani S.G. (Ed) "Flow and rheology in polymer composites manufacturing", 1994, Elsevier Science B.V.

Racineux G., "Rhéologie des pâtes minérales: cas du mélange $\mathrm{TiO}_{2}-\mathrm{HNO}_{3}$ destiné à l'extrusion de supports de catalyseur", Thèse de doctorat l'Ecole normale supérieure de Cachan, 1999.

Yaguchi H., Hojo H., Lee D.G., Kim E.G., "Measurement of planar orientation of fibers for reinforced thermoplastics using image processing", Intern. Polymer Processing, vol. 3, 1995, p. 262-269. 